\title{
Review of The Interpersonal Communication Book, 13th edition by Joseph A. Devito, New York: Pearson, 2012, 432 pages
}

The topic of interpersonal communication has recently gained increasing acknowledgement as a separate field of study in the broader family of communication studies, in a change of perspective that now takes our daily one-on-one interactions into account as the relevant, micro-scale acts that represent the foundation of all larger-scale communication - be it corporate, mass media, or online social media.

Despite numerous handbooks, encyclopaedias and essential volumes on the topic which became available on the market throughout the years, one book stands apart, and has now reached its $13^{\text {th }}$ edition and continues to inspire and inform young academics in universities worldwide. Joseph A. Devito's “The Interpersonal Communication Book" is a well-structured and fundamental book on interpersonal communication, which covers topics as broad as relationships, conflict and communication ethics, and proves the key point that interpersonal communication is many-faceted, depending on dimensions such as individual apprehension, assertiveness or adequacy. We cannot speak of a general thesis that the book defends, but rather of separate conclusions for each unit, ranging from the idea that self-disclosure in a relationship at its early stages is perceived as riskier by women, as compared to men (in the part dedicated to the self in communication), to newly found support for the theory that all verbal and nonverbal communication is intentional and a matter of choice.

This updated book appears to be a truly complete view of the literature and research in the field of interpersonal communication. At the same time it is a very practical compendium, as it openly offers suggestions for effective communication when considering ethics, diversity and power in interpersonal communication, as well as differentiating between face-to-face and mediated (computer-mediated) interaction.

Thus, most noteworthy, the $13^{\text {th }}$ edition of the book is brought up to date and adjusted to strongly highlight the concept of choice in communication. Devito clearly stresses how to adapt our skills in order to make better choices in communication, irrespective of the context (personal, social, professional) and, at the same time, directs readers towards bettering their social interactions through a rich palette of interpersonal skills. There is also an updated focus on culture and interpersonal communication, with aspects of cultural diversity integrated in all major units of the book.

The author successfully and diligently walks the readers through the specifics of interpersonal communication, and demonstrates that it is a process through which individuals define themselves and their interaction partners, through ever-changing and periodically redefined

* PhD student, Communication Science, National University of Political Studies and Public Administration, Bucharest, Romania, ioana.schiau@comunicare.ro. 
relationships. The book is well supported by the creative use of literature and detailed evidence of studies and research, which makes it a significant contribution to the field.

"The Interpersonal Communication Book" is organized in separate parts and subunits, addressing key aspects of interpersonal communication, such as the Preliminaries to Interpersonal Communication, Interpersonal Messages, Interpersonal Relationships, each separate part being a comprehensive theoretical review.

Within the first part, a unit that is worth reading is "Perception and the Self and Others in Interpersonal Communication", dwelling on such specific dimensions as self-disclosure and "impression management", testing students to see how accurate they are at people perception.

The second part of the book is structured to deal with verbal, nonverbal, emotional and conversational messages. In terms of verbal messages, special attention should be paid to the subunits discussing ethics in communication and lying, in particular. The section on nonverbal communication guides readers through the main principles of nonverbal communication and ends with an interesting test for students -"Do you avoid touching?" which should perhaps, more effectively than any theory cited, prove to students that there are very different nonverbal customs in the world.

The book makes use of charts, graphs and photographs that contribute to the clarity of the information provided, while at the same time it prompts readers to make comparisons and perhaps find valuable new research questions of their own. Such examples are the graphs detailing "body availability" (areas where touching is permitted) for male and female college students in the USA (S.M. Jourard) and "areas and frequency of touching" in Japan versus the USA (Dean C. Barnlund), in the unit on nonverbal communication.

The book seems to differ in structure from other similar works in the field - as Kory Floyd's "Interpersonal Communication" (Floyd, 2013), to name just one of the books that have recently entered the Romanian market - in the sense that it has a more organic view on matters. There is a difference in approach which can be noticed by simply looking at the table of contents. While Floyd's book seems to have a slightly more scholarly (verging on arid) notion on how to organize its information, Devito's book is intuitively and psychologically ordered. Parts and units such as "perception, the self and others", "emotional messages", "interpersonal conflict and conflict management", "interpersonal relationships", which allow for a clear and easy to memorise overview of all the dimensions of interpersonal communication.

The chapter on the self raises questions about self-disclosure, which is ultimately presented as a matter of choice. In fact, the concept of choice is presented in a variety of contexts, demonstrating that there are right and wrong decisions when it comes to developing a communicational relationship, ranging from the conscious choice of a "life position" (such as the "I'm OK, you're OK" script), to decide how to respond to a partner's silence.

In the updated version, each new chapter opens with Interpersonal Communication Choice Point Videos (which students can access online, on MyCommunicationLab.com). The information in the section is presented as a related scenario which students can follow and afterwards observe different endings for the scenario, to see how their different choices end. In terms of new didactic instruments used, the updated version of the book has clearly become even more interactive, containing side boxes (such as the "Ethics in Interpersonal Communication" boxes, containing "Ethical Choice Points") that encourage students to think how they would act in day-to-day situations.

A truly interesting subunit of the book analyses listening and silence - in other words, the things we tell each other when we do not speak. Devito makes clever use of literature to point out that 
silence is an aspect that we can connect to nonverbal communication, and which can be seen dichotomously, either as creating walls and isolation, or as serving a complete and independent function in itself, while listening is a skill that is a must for communicational proficiency.

The book also addresses the very opposite of silence, namely the human voice and impression forming based on one's tone and voice volume. It is, thus, a paralanguage cue that Devito analyses, and cites studies that prove that we are able to judge age, sex and, most surprisingly, even social status (upper, middle, lower class) with remarkable accuracy. However, studies cited prove that, when attempting to ascertain one's personality based solely on paraverbal, voice interpretation, we are often in error - thus once more raising questions about subjectivity and its interference with our power of analysis.

However, since this is ultimately an all-comprehensive textbook, it does not extensively explain the interconnections of all the aspects, triggers, contexts and effects of interpersonal communication. There are, nonetheless, indicators of how each unit connects to the next, in practical terms.

For instance, in the part covering nonverbal communication we find indicators of how nonverbal behaviour connects with status roles and power indicators. Touching is, in most cultures, dependent on status, determined by what Nancy Henley (cited in the book) calls Body Politics, wherein those with higher power status can touch those with lower power status and not vice versa. Conversely, in the part covering issues of interpersonal relationships, we find a unit dedicated to power in interpersonal relationships, which details the power aspect that all relationships have and the "power play" we all engage in.

The $13^{\text {th }}$ edition is also available in an innovative physical format of an unbound binder (and thus allows students to integrate their own notes and bring to class only the needed sections). Despite the fact that it has become a classic guide to interpersonal communication, one new edition after another, the book does risk becoming engulfed by the very generous offer of textbooks on the subject that are available in bookstores and libraries. The next editions must continue the clear line of innovation of the $13^{\text {th }}$ reprint, in order for the book to remain one of the best ranked textbooks.

\section{Reference}

1. Floyd, K. (2013), Comunicarea Interpersonală, Iași: Polirom. 